\title{
Tratamento de Taquicardia Ventricular Induzida por Mola de Choque de Eletrodo de Cardioversor Desfibrilador Implantável
}

Treatment of Ventricular Tachycardia induced by Coil of Ventricular Lead of Implantable Cardioverter Defibrillator

William Oliveira de Souza ${ }^{1, *}$, Pablo Ferreira Reis ${ }^{1}$, Fábio Lopes Erthal', Rodrigo Minati Barbosa'

ORCID IDs

Souza WO (iD https://orcid.org/0000-0001-7828-1867

\section{RESUMO}

Paciente portador de cardioversor desfibrilador implantável (CDI)-DDD por cardiopatia arritmogênica de etiologia desconhecida, com indução de taquicardia ventricular pela mola de ventrículo direito (VD) do eletrodo de choque. A arritmia gerada pelo próprio eletrodo do CDI foi causa de múltiplos episódios com terapia apropriada por anti-tachycardia pacing (ATP) e choque. Confirmada a etiologia da arritmia por estudo eletrofisiológico e realizado tratamento bem-sucedido com ablação, sem necessidade de reposicionamento cirúrgico do eletrodo.

PALAVRAS-CHAVE: Arritmias cardíacas; Taquicardia ventricular; Desfibriladores implantáveis; Eletrodos implantados; Ablação por cateter; Complicações pós-operatórias.

\section{ABSTRACT}

Implantable cardioverter defibrillator (ICD) -DDD for arrhythmogenic heart disease of unknown etiology, with the induction of ventricular tachycardia by the right ventricle (RV) of the shock electrode. The arrhythmia generated by the ICD electrode itself was the cause of multiple episodes with appropriate anti-tachycardia pacing (ATP) and shock therapy. The etiology of the arrhythmia was confirmed by electrophysiological study and successful treatment was performed with ablation, without the need for surgical repositioning of the electrode.

KEYWORDS: Cardiac arrhythmias; Ventricular tachycardia; Implantable defibrillators; Implanted electrodes; Catheter ablation; Postoperative complications. 


\section{INTRODUÇÃO}

Relata-se caso interessante pela indução de arritmia ventricular pelo próprio dispositivo que visa tratá-la. O tratamento foi feito com ablação por cateter de radiofrequência, sem necessidade de reposicionamento cirúrgico do eletrodo.

\section{MÉTODOS}

O paciente é acompanhado no Serviço de Arritmia do Instituto Nacional de Cardiologia, estado do Rio de Janeiro. O caso foi montado com revisão dos registros de prontuário, dos estudos eletrofisiológicos e anamnese. Realizada revisão bibliográfica ${ }^{1-3}$. Pesquisa na base de dados do PubMed com os termos "implantable cardioverter defibrillator lead complications", "icd lead replacement", "icd lead induced arrhythmia", "icd coil induced arrhythmia” não retornou casos semelhantes até 05/04/2018.

\section{RELATO DO CASO}

Paciente ACJ, masculino, 45 anos em 2010, atendido em unidade de pronto atendimento pública com quadro de taquicardia ventricular monomórfica sustentada (TVMS) e instabilidade hemodinâmica, sendo tratado com cardioversão elétrica (CVE) e o evento classificado como análogo à morte súbita abortada. Encaminhado para serviço especializado do Sistema Único de Saúde (SUS). Em abril de 2011, foi atendido no Serviço de Arritmia do Instituto Nacional de Cardiologia, sendo internado para investigação adicional. Os exames da ocasião evidenciaram ecocardiograma com ausência de cardiopatia estrutural, coronariografia normal, teste ergométrico com bigeminismo ventricular ao pico do esforço (9,6 mets) e dois exames de holter $24 \mathrm{~h}$ com vários episódios de taquicardia ventricular não sustentada (TVNS). Optou-se por estudo eletrofisiológico com reindução de TVMS, ciclo de 290 ms (207bpm) inicialmente estável, havendo aceleração e instabilidade após tentativa de estimulação anti-taquicardia ("antitachycardia pacing" ou ATP) com necessidade de CVE. $\mathrm{Na}$ mesma internação foi submetido à implante de cardioversor desfibrilador implantável (CDI)-DDD com eletrodo de choque de dupla mola (unidade geradora Biotronik Lumax 340 DRT, eletrodo de choque Biotronik Linox SD 65/16, eletrodo de átrio Briotronik Setrox S53). Em dezembro de 2011, o paciente reinternou com quadro de tempestade elétrica por múltiplas TVMS. Interrogação do dispositivo demonstrou que todas as terapias foram apropriadas. Após ajuste de drogas e cessação das arritmias, o paciente recebeu alta hospitalar. Questionou-se, na época, a possibilidade de um estudo eletrofisiológico e ablação (EEF/ABL), dependendo de avaliação clínica posterior. Porém, evoluiu sem novas arritmias até esgotamento da unidade geradora em 2014, sendo submetido à troca dessa (Medtronic Virtuoso II DR), mantendo-se os eletrodos.

Em fevereiro de 2017, o paciente procurou atendimento por um choque. Interrogação do dispositivo evidenciou cerca de 200 episódios de taquiarritmia, a maioria de TVNS, a minoria de TVMS interrompida com ATPs e um episódio com falha em sucessivos ATPs e choque apropriado. Marcado novo EEF/ABL em uma semana e ajustadas drogas, porém o paciente evoluiu sem nenhum episódio de arritmia, sendo cancelado o procedimento para observação.

Em agosto do mesmo ano, o paciente retornou com >2.000 episódios de TVNS e TVMS. Todas as arritmias sustentadas foram interrompidos com ATPs. Novo ecocardiograma mantinha ausência de cardiopatia estrutural identificável. Submetido então ao EEF/ABL com mapeamento eletroanatômico. Anteriormente ao procedimento, as terapias do CDI foram desativadas e a unidade reprogramada para modo VVI $30 \mathrm{bpm}$ (supressão de estimulação), estando o paciente em ritmo sinusal durante todo o procedimento. Mapa de voltagem evidenciou ausência de cicatrizes endocárdicas. O EEF induzia com facilidade TVNS e TVMS, com ciclo de $315 \mathrm{~ms}$ (190 bpm), estabilidade hemodinâmica e interrupção com ATP. Observada ao mapeamento eletroanatômico região de maior precocidade anexa à porção proximal da mola de choque de VD. Nessa região, observou-se precocidade, potencial mesodiastólico e semelhança de 12/12 na manobra de pace mapping. Determinada essa região como o foco da arritmia apresentada, sendo realizadas aplicações de radiofrequência justapostas à mola de choque endocardicamente em ambos os lados dessa como "trilho", com maior concentração de aplicações no aspecto medial e proximal da mola (Fig. 1). Após 
o fim das aplicações, não mais foi induzida arritmia ventricular. Ao fim do procedimento, interrogação do CDI demonstrou estabilidade da impedância do eletrodo de ventrículo direito (VD) e mola de choque, sugerindo que a aplicação de radiofrequência, embora justaposta, não incutiu dano ao eletrodo. Retornada a programação para a anterior ao procedimento. Evoluiu com estabilidade, recebendo alta após $48 \mathrm{~h}$ do procedimento em uso de amiodarona $200 \mathrm{mg} / \mathrm{dia}$, bisoprolol $10 \mathrm{mg} / \mathrm{dia}$ e ramipril $2,5 \mathrm{mg} /$ dia. Retorno em uma semana não apresentou novas arritmias. Última avaliação em setembro de 2017, sem novos episódios de arritmia (Tabela 1).

\section{DISCUSSÃO}

O implante de dispositivos eletrônicos cardíacos está em expansão, evidenciando novos desafios na prática clínica. A presença de arritmias no pós-operatório de implante de dispositivos eletrônicos implantáveis cardíacos é conhecida de longa data e aguarda-se melhora dessas em 48 h após o procedimento. ${ }^{4}$ Em experiência anterior do Instituto Nacional de Cardiologia, já foi observada TVMS em eletrodo de choque de CDI, porém com origem na ponta do eletrodo ${ }^{5}$, local de conhecida fibrose e possíveis complicações arritmogênicas. $\mathrm{O}$ caso apresentado

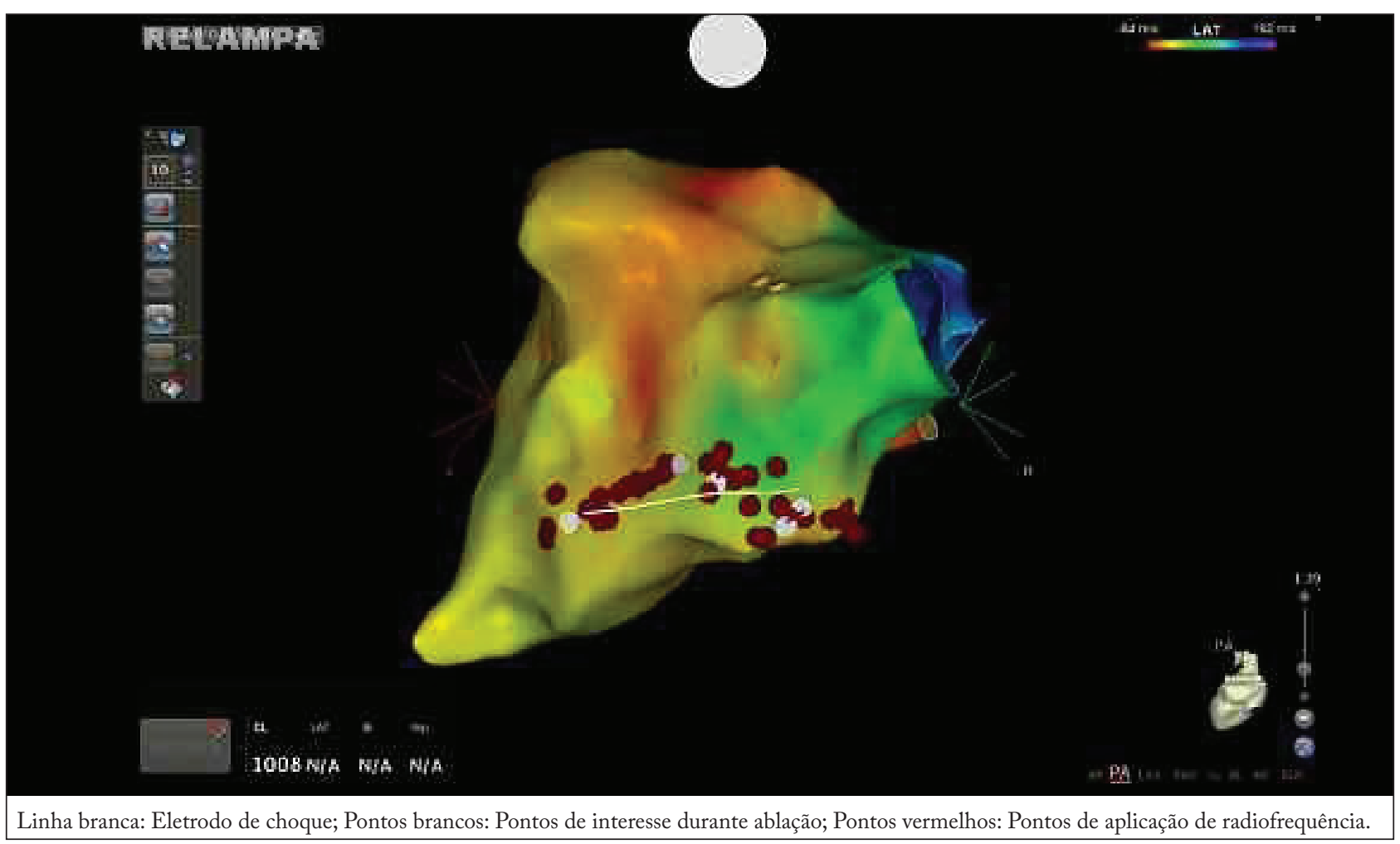

Figura 1. Mapeamento eletroanatômico em ritmo sinusal.

Tabela 1. Interrogação do CDI, ablação em 18 de agosto de 2017.

\begin{tabular}{|c|c|c|c|c|c|}
\hline Eventos & $\begin{array}{c}26 \text { Maio } 2017 \\
\text { a } \\
28 \text { Julho } 2017\end{array}$ & $\begin{array}{c}28 \text { Julho } 2017 \\
\text { a } \\
18 \text { Agosto } 2017\end{array}$ & $\begin{array}{c}18 \text { Agosto } 2017 \\
\text { a } \\
25 \text { Agosto } 2017\end{array}$ & $\begin{array}{c}25 \text { Agosto } 2017 \\
\text { a } \\
29 \text { Setembro } 2017\end{array}$ & $\begin{array}{l}\text { Total desde } \\
8 \text { Julho } 2017\end{array}$ \\
\hline Fibrilação ventricular & 0 & 0 & 0 & 0 & 0 \\
\hline $\begin{array}{l}\text { Zona de taquicardia } \\
\text { ventricular rápida }\end{array}$ & 0 & 0 & 0 & 0 & 26 \\
\hline Taquicardia ventricular & 43 & 114 & 0 & 0 & 247 \\
\hline $\begin{array}{l}\text { Taquicardia } \\
\text { ventricular não } \\
\text { sustentada }\end{array}$ & 2033 & 2817 & 0 & 0 & Dado indisponível \\
\hline $\begin{array}{l}\text { Anti-tachycardia } \\
\text { pacing }\end{array}$ & 43 & 114 & 0 & 0 & 272 \\
\hline Choques & 0 & 0 & 0 & 0 & 1 \\
\hline
\end{tabular}


é relevante pela documentação de arritmia ocasionada pelo contato da mola de choque com o endocárdio. A presença da precocidade ao mapeamento eletroanatômico, bem como o tratamento bem-sucedido com ablação na região alvo, fortemente sugere a origem dessa arritmia na mola de choque de VD. Não está claro se a arritmia, nesse caso, foi causada apenas pela presença do contato da mola de choque com o endocárdio ou se a condição arritmogênica prévia do paciente contribuiu para sua indução, posto que não foi definida a etiologia da arritmia que levou ao evento índice em 2010 e a tempestade elétrica em 2011. Importante salientar que as morfologias das TVMS obtidas nos EEF de 2011 e 2017 são díspares em ciclos e morfologias, sugerindo focos afastados.
A ablação por cateter de radiofrequência foi efetiva em suprimir a ocorrências de repetidos episódios de TVNS e terapias inapropriadas, sem necessidade de reposicionamento cirúrgico do eletrodo de choque.

\section{AGRADECIMENTOS}

Ao professor Bernardo Rangel Tura, pela revisão do artigo.

\section{CONTRIBUIÇÃO DOS AUTORES}

Todos os autores contribuiram igualmente para este artigo.

\section{REFERÊNCIAS}

1. Ellenbogen KA, Neal Kay G, Lau CP. Clinical cardiac pacing, defibrillation, and resynchronization therapy. 3a ed. Philadelphia: Saunders; 2007.

2. Melo CS. Tratado de estimulação cardíaca artificial. 5a ed. Tamboré: Manole; 2015.

3. Al-KhatibSM, Stevenson WG, Ackerman MJ, BryantWJ, CallansDJ, Curtis AB, et al. 2017 AHA/ACC/HRS guideline for management of patients with ventricular arrhythmias and the prevention of sudden cardiac death. Circulation. 2018;138(13):e272-e391. https://doi.org/10.1161/CIR.0000000000000549

4. Brito Junior HL, Gauch PRA, Oliveira AS de. Arritmias induzidas por marcapasso cardíaco. JBAC. 1990;3(3):88-93.

5. Souza WO, Seifert MMS, Pinho DL, Nascimento EAD, Saad EB, et al. Anais do $28^{\circ}$ Congresso de Cardiologia da Socerj; 3-5 de Agosto de 2011; Rio de Janeiro. 